\title{
Multi-pass Mapping Schemes for Parallel Sparse Matrix Computations ${ }^{\star}$
}

\author{
Konrad Malkowski and Padma Raghavan \\ Department of Computer Science and Engineering, \\ The Pennsylvania State University, \\ 343K IST Building, University Park, PA 16802-6106 \\ \{malkowsk, raghavan\}@cse.psu.edu
}

\begin{abstract}
Consider the solution of a large sparse linear system $A x=b$ on multiprocessors. A parallel sparse matrix factorization is required in a direct solver. Alternatively, if Krylov subspace iterative methods are used, then incomplete forms of parallel sparse factorization are required for preconditioning. In such schemes, the underlying parallel computation is tree-structured, utilizing task-parallelism at lower levels of the tree and data-parallelism at higher levels. The proportional heuristic has typically been used to map the data and computation to processors. However, for sparse systems from finite-element methods on complex domains, the resulting assignments can exhibit significant load-imbalances. In this paper, we develop a multi-pass mapping scheme to reduce such load imbalances and we demonstrate its effectiveness for a test suite of large sparse matrices. Our scheme can also be used to generate improved mappings for tree-structured applications beyond those considered in this paper.
\end{abstract}

\section{Introduction}

Many computational science and engineering applications concern the numeric solution of models based on nonlinear partial-differential-equations on complex domains, which are discretized using finite-element or finite-difference methods. When implicit or semi-implicit schemes are used in the solution process, the total application time can be dominated by the time required for the solution of the underlying sparse linear systems of the form $A x=b$. Consequently, effective parallel sparse linear system solution is of critical significance in such large-scale applications.

A solution to $A x=b$, where $A$ is sparse, can be achieved using either direct methods or preconditioned iterative methods. In parallel sparse direct solvers,

\footnotetext{
* The work was supported in part by the National Science Foundation through grants NSF ACI-0102537 and NSF CCF-0444345, and by the Director, Office of Science, Division of Mathematical, Information, and Computational Sciences of the U.S. Department of Energy under contract number DE-AC03-76SF00098.
} 
a Cholesky $\left(A=L L^{T}\right)$ or an $\mathrm{LU}(A=L U)$ factorization is first computed and then used for triangular solution [1,2, 4, 6, 8, 16]. For preconditioning, incomplete counterparts of both types of factorizations can be utilized to compute a sparse approximation to the factors to accelerate the convergence of an iterative method such as Conjugate Gradients or GMRES [7,10,17, 18, 19. Efficient implementations on distributed memory multiprocessors require data and task assignments that can balance the computational load for the factorization step among processors. The computations in the factorization step are tree-structured and are formulated bottom-up on a supernodal tree using either effectively dense panels of columns in a left-looking panel scheme [1] or using dense triangular matrices in a multifrontal scheme $8,9,13,16$.

In this paper, we focus on mapping tree-structured computations typical of sparse factorizations where assignments generated by the popular proportional mapping [5, 15] scheme often exhibit large imbalances. Our main contribution is the formulation of a new multi-pass refinement scheme that can substantially improve the quality of the assignment and thus the performance of parallel factorization codes. In the next section, we provide a brief review of parallel sparse factorization. In Section 3, we begin with a review of the original proportional mapping scheme [15] and then describe our new multi-pass schemes. In Section 4, we provide an empirical evaluation of the performance of our multi-pass schemes and the original proportional mapping. In Section 5, we summarize our contributions and discuss further extensions and applications.

\section{Parallel Tree-Structured Sparse Factorization}

Sparse matrix factorization and its incomplete variations typically require a four step process: (1) ordering to compute a fill-reducing numbering, (2) symbolic factorization to determine the nonzero structure of the factor, (3) numeric factorization, and, (4) triangular solution. The first ordering step is also critical for determining the parallelism and the total computational costs over all remaining steps. A well-established practice is to compute orderings, using for example, nested dissection techniques that recursively partition the graph of $A$ using vertex separators 3, 11. After this step, the parallelism available for the subsequent factorization and triangular solution step can be represented by a tree. This tree can be weighted to represent computation costs and the tree can be mapped to processors to enable load-balanced computation of the factorization step.

We provide a brief overview of parallel sparse Cholesky factorization using a small example to illustrate the main ideas, which are described in greater detail in the survey article by Heath et al. 8]. Figure 1 concerns the sparse matrix $A$ of a five-point $7 \times 7$ finite-difference grid, which is widely used as a model problem in this area. The columns of the Cholesky factor $L$ of this matrix, can be grouped into supernodes [12. A supernode is a set of consecutive columns that have nested sparsity structure, and can essentially be treated as a dense block, see for example, the last seven columns in Figure 1. As a consequence of sparsity in $L$, columns in a supernode need not be updated by columns in all 

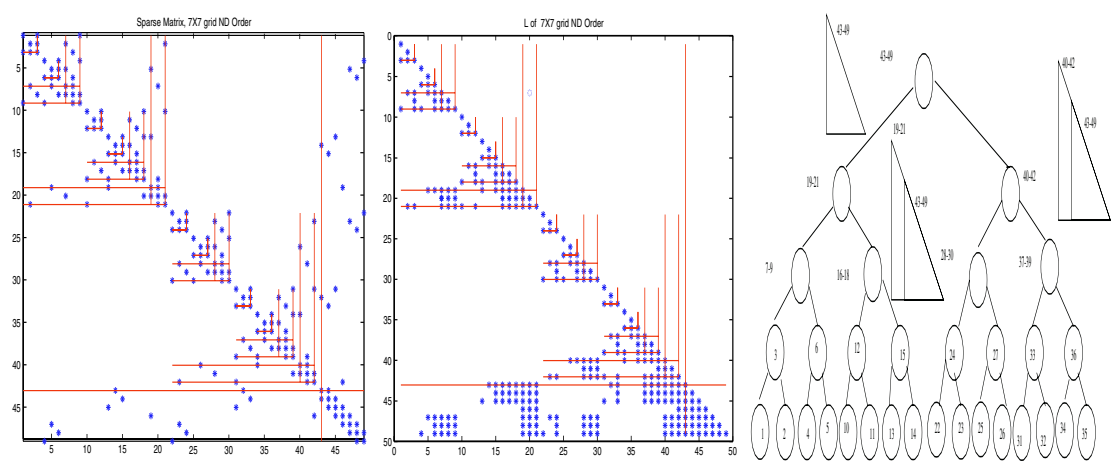

Fig. 1. The structure of a sparse matrix $A$ from a $7 \times 7,5$-point finite-difference grid reordered to reduce fill (left), the structure of $L$ shown with a recursive partition (middle) and a multifrontal scheme on the binary supernodal tree (right)

preceding supernodes in the numeric factorization, instead columns in a supernode $v$ are updated only by columns in supernodes within the subtree rooted at $v$ [12. The sparsity structure of $L$ can be viewed in terms of effectively dense column-blocks or alternatively, in a recursive manner in terms of submatrices, as shown in Figure 1. The two types of cache-efficient numeric factorizations are a column-block scheme [14] and a multifrontal scheme [2]. The two schemes differ in how they compute and apply updates to columns in a given supernode from columns in earlier supernodes. In a multifrontal scheme, dense triangular matrix operations are used to factor the columns in a supernode and to accumulate and propagate updates from these columns to those at the parent and ancestor supernodes, as shown in Figure 1. Multifrontal schemes typically lead to efficient parallel implementations [6] 16].

Parallel implementations of both left-looking or multifrontal factorization depend on the supernodal tree which can be weighted to represent the corresponding computation and communication costs [5, 6, 16]. For illustrative purposes, view this tree as a complete binary tree with more leaves than the number of processors $P$ and with all nodes having the same computational cost. Now, at some level $l=\log _{2} P, P$ disjoint nodes can be identified and the subtrees rooted at these nodes can be assigned to distinct processors. These subtrees represent disjoint local computations at processors with ideal task-parallelism. At a level higher than this one, disjoint processor groups of size 2 can cooperate to perform data-parallel computations at the supernode and so on, until all processors participate at the root. This is known as the balanced subtree to processor mapping. The proportional mapping scheme is a generalization of this scheme to derive assignments for practical problems where the supernodal tree can be highly irregular and the computation at a node and total computations in subtrees can vary dramatically. 


\section{Multi-pass Mapping Schemes}

In this section, we begin with an overview of the original proportional mapping scheme [15] and continue with the formulation of our multi-pass assignment schemes. The latter seek to refine and improve an assignment obtained from the proportional mapping, which is used in the first step.

To provide a precise statement of our mapping schemes, we start with a definition of the weighted supernodal tree and a valid mapping, i.e., an assignment of computations represented by the tree to a set of processors. Consider a supernodal tree $T(r)=(V, E, N W, S W)$ with $V$ vertices, $E$ edges, rooted at $r \in V$ with two weighting functions $S W$ and $N W$ representing a suitable measure of computational costs. For each vertex $v \in V, N W(v)$ is the nodal weight, corresponding to the cost of computations at the node $v$. The subtree weight of $v, S W(v)$ is defined as the sum of nodal weights of all vertices in $T(v)$, the subtree rooted at $v$. A mapping $M=(T, P)$ indicates an assignment of a set of $P$ disjoint subtrees $T\left(v_{0}\right), T\left(v_{1}\right), \cdots T\left(v_{p-1}\right)$ (including all leaf vertices in $V$ ) to processors $0,1, \cdots(P-1)$. These disjoint subtrees represent local task parallel computations; computations at an interior vertex $v$ are shared equally among all processors assigned subtrees in $T(v)$. However, such computation cannot proceed until all processors can synchronize at the internal node. Consequently, load imbalances among processors along different paths leading to a vertex $v$, result in some processors remaining idle until all can synchronize and proceed with the computations at $v$.

The proportional mapping [15] is a recursive scheme with an initial assignment of $P$ processors to the root $r$ and thus $T(r)$. Consider $T(v)$ the subtree at $v$, which has been assigned $p$ processors. If $p=1$, the recursion terminates; otherwise for $p>1$, for each child $c$ of $v$ assign $p_{c}$ to $T(c)$ where $p_{c}=p \times \frac{S W(c)}{S W(v)-N W(v)}$. Some rounding scheme must be used to ensure that $p_{c}$ is an integer number of processors and that $\sum_{c,(c, v) \in E} p_{c}=p$. We experimented with several rounding schemes to select the one that leads to the best mappings for our test collection. In this scheme, at a vertex $v$ with $T(v)$ assigned $p$ processors, we compute for each child $c, \hat{p}_{c}=\left\lfloor p \times \frac{S W(c)}{S W(v)-N W(v)}\right\rfloor$, and the projected load $\hat{W}(c)=\frac{S W(c)}{\hat{p}_{c}}$. Next, we compute $\tilde{p}=p-\hat{p}$ where $\hat{p}=\sum_{c,(c, v) \in E} \hat{p}_{c}$. Let $c_{1}, c_{2}, \cdots c_{\tilde{p}}$ be the children vertices of $v$ with the $\tilde{p}$ highest values of the projected load $\hat{W}\left(c_{i}\right)$; for these vertices we set $p_{c} \leftarrow \hat{p}_{c}+1$ while for the others, we set $p_{c} \leftarrow \hat{p}_{c}$. Our multi-pass assignment scheme uses this proportional mapping as the first step.

Rounding effects, incurred to ensure an integer number of processors at each node, can be exaggerated by irregular subtree weights resulting in assignments with processor loads that are not well-balanced. Consequently, the highest load at a processor, i.e., the critical path weight, can be substantially higher than the ideal of $S W(r) / P$ as shown in the next section in Figure 2

Our multi-pass schemes attempt to refine the assignment from the proportional mapping in order to improve the worst load at a processor. We therefore start with a precise definition of this quantity. Consider any mapping $M=$ $(T(r), P)$ of the supernodal tree $T(r)=(V, E, N W, S W)$ with disjoint sub- 

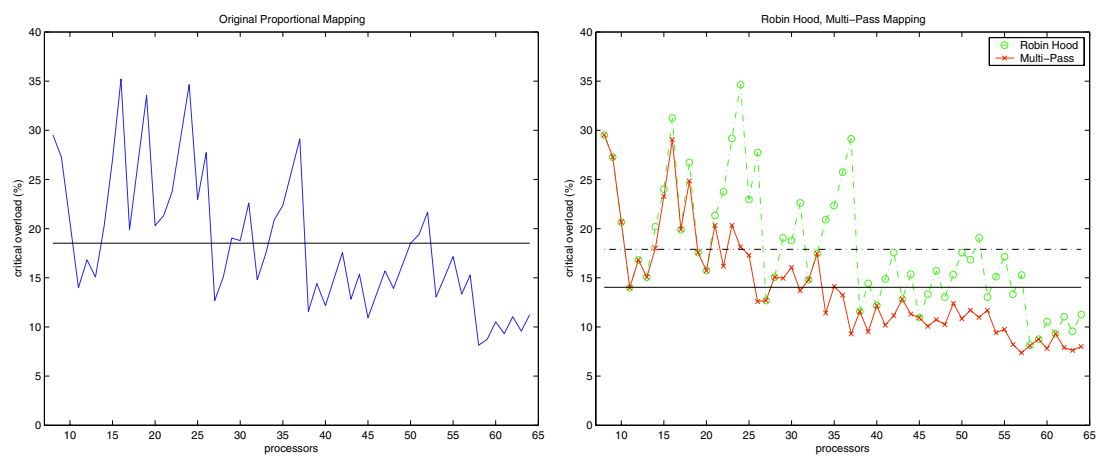

Fig. 2. Critical overload for the original proportional heuristic (left) and and the Robin Hood and multi-pass schemes (right) for augustus7 on $8-64$ processors

trees $T\left(v_{0}\right), T\left(v_{1}\right), \cdots T\left(v_{P-1}\right)$ assigned to processors $0,1, \cdots(P-1)$. Let $\pi(v)$ denote the number of processors assigned to a node $v$; note that $\pi(r)=P$. Let $\operatorname{path}(i)$ denote the intermediate vertices in $T$ from the parent of $v_{i}$ to the root $r$. Now the workload of a processor $p_{i}, 0 \leq i \leq(P-1)$ is given $W\left(p_{i}\right)=S W\left(T\left(v_{i}\right)\right)+\sum_{v \in \operatorname{path}\left(v_{i}\right)} N W(v) / \pi(v)$. An ideal assignment would lead to the ideal load $I(M)=S W(r) / P$. The heaviest load at a processor, which corresponds to a critical path is given by $H(M)=\max _{p_{i}, 0 \leq i \leq P-1}\left\{W\left(p_{i}\right)\right\}$; likewise, we define the lightest load $L(M)=\min _{p_{i}, 0 \leq i \leq P-1}\left\{\bar{W}\left(p_{i}\right)\right\}$; Our goal is compute mappings where $H(M)$ is close to $I(M)$ for a given $T$ and $P$.

We next present two refinement schemes, followed by our final multi-pass scheme which enables their effective combination.

A Robin Hood Refinement Scheme. Assume that an assignment $M$ has been provided either from proportional mapping or the application of one or more refinement schemes. First, determine $H(M)$ and $L(M)$ corresponding to the heaviest and the lightest loads at a processor. Let $p_{h}$ and $p_{l}$ denote the corresponding processors assigned to subtrees $T(h)$ and $T(l)$, rooted at vertices $h$ and $l$ respectively. We consider $p_{h}$, the overworked processor to be "poor "and the lightly loaded $p_{m}$ to "rich." Our Robin Hood scheme, removes a processor assigned along the lightest path, path $(l)$ and uses it to refine the mapping of the local subtree $T(h)$ and thus reduce the load along critical path path $(h)$. The Robin Hood scheme is typically applied four times to allow refinement of paths with weights close to the weight of the critical path, and the best assignment is retained.

Iterative Correction with Processors in Reserve. Assume we are seeking an assignment $M=(T(r), P)$ with $P$ processors. We first obtain a mapping with $\tilde{M}=(T(r), \tilde{P})$ with $\tilde{P}$ processors where $\tilde{P}<P$. Let $\hat{P}=P-\tilde{P}$ denote the remaining processors "held in reserve." Our correction algorithm proceeds thus in $\hat{P}$ iterations, starting with a mapping $M_{1}$ initialized to $\tilde{M}$ with $P_{1}=\tilde{P}$ processors. At iteration $i$, compute $H\left(M_{i}\right)$ and let $p_{h}$ be the corresponding processor with the 
heaviest load. Refine the mapping of $T(h)$ by adding another processor to vertex $h$ to obtain a new mapping $M_{i+1}$ with $P_{i+1}=P_{i}+1$ processors. A disadvantage of this scheme is that improvements depend on the initial choice of $\tilde{P}$.

A Multi-pass Mapping Scheme. We now combine the two refinement schemes with the original proportional mapping to present our final multi-pass mapping scheme. The first pass is the proportional mapping scheme. In the second pass, this mapping is refined using the Robin Hood scheme. Let this result in a mapping $\hat{M}$ with the specified number of processors, $P$. Compute $H(\hat{M})$; if this quantity is more than the ideal load $(S W(r) / P)$, then compute $\tilde{P}=S W(r) / H(\hat{M})$, where $\tilde{P}<P$. Apply the proportional mapping to obtain a mapping $\bar{M}$ with $\tilde{P}$ processors. Refine it using the Robin Hood scheme to obtain a new mapping $\tilde{M}$ with $\tilde{P}$ processors. Finally, refine this mapping using iterative correction with $\hat{P}=P-\tilde{P}$ processors in reserve. This defines our overall multi-pass mapping scheme.

\section{Empirical Results}

In this section, we empirically evaluate the quality of assignments for performing parallel sparse Cholesky factorization. We report on the improvements observed when our schemes are used to refine the assignments computed by the original proportional scheme. We use a collection of well-known sparse matrices from finite-element analysis of three dimensional structures and shells, and one problem from computational fluid dynamics.

Our test suite of matrices and best observed improvements are shown in Table 1. We consider the factorization of these matrices using 8-64 identical pro-

Table 1. Description of test matrices, relative critical loads (RCL), and numeric factorization times using the original proportional scheme and the multi-pass scheme. Each matrix - processor pair corresponds to the best observed improvements of the RCL metric using our multi-pass scheme. The column labeled "Error" indicates the difference between predicted and observed execution times

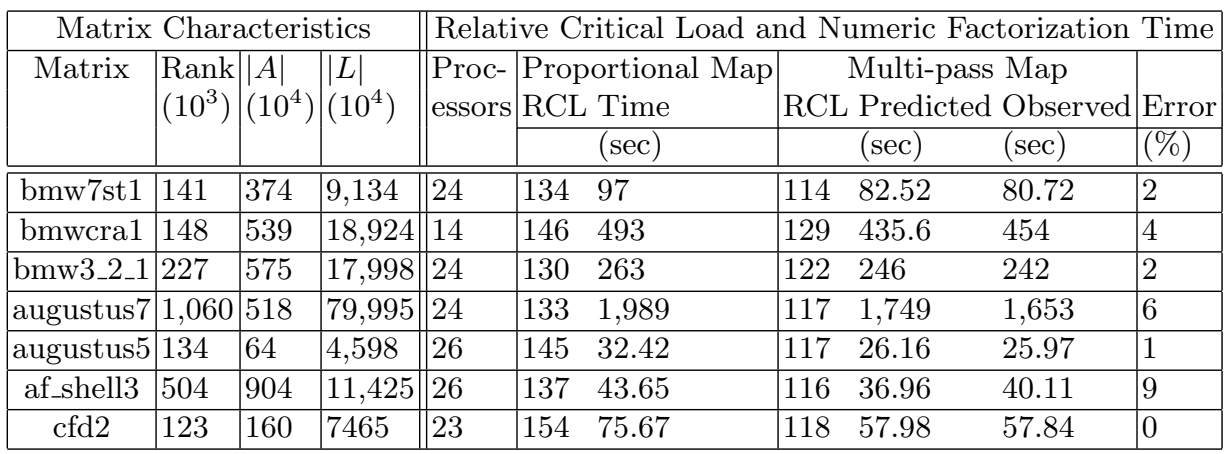


cessors after ordering using a nested dissection scheme. The supernodal trees were weighted to represent computational costs (for floating point operations, and not for communication or for other integer operations) in a parallel multifrontal scheme, for example, such as the scheme in the DSCPACK software [16].

Our results concern the quality of mappings as defined by the heaviest workload at a processor, i.e., the critical path weights. Consider $T(r)$ corresponding to a specific problem. For each mapping $M$ of $T(r)$ using some $P$ processors in the range $8-64$, we focus on heaviest load at a processor, i.e., the critical path cost, as the main metric indicative of the quality of the mapping. The closer this metric, $H(M)$ (defined in the earlier section) is to the ideal load $(I=S W(r) / P)$, the better is the quality of the mapping. Over the range of processors, problems and mapping the actual value of this metric can vary significantly making direct comparisons difficult. We therefore use the following two scaled forms: (i) the relative critical load, (RCL) defined as $\frac{H(M)}{I} \times 100$, and (ii) the critical overload, (CO) defined as $\frac{H(M)-I}{I} \times 100$.

We begin with some experiments (reported in the right half of Table1) to verify that the relative critical load (and the critical overload) metric corresponds well to the actual performance of the numeric factorization step. We used the DSCPACK software [16] with two different mappings, one from the original proportional scheme and the other from our multi-pass scheme. Our experiments were performed on a cluster with 81 dual-processor compute nodes with AMD Athlon MP2200+ processors, with 71 nodes having 1 GB of main memory, and 10 nodes having $2 \mathrm{~GB}$ of main memory, and a $9 \times 9$ torus Scali interconnect. Table 1 shows the relative critical load (RCL) and CPU time for numeric factorization using the original proportional mapping. We then compute assignments using our multi-pass scheme and their corresponding values of the relative critical load (also reported in Table 1). Using the relative critical load metrics for the original and multi-pass mappings and the CPU time for numeric factorization with the proportional mapping, we can project the estimated numeric factorization time for the new assignment (reported in the column labeled "Predicted" time). We next performed numeric factorization with the new assignments and observed actual CPU time. As shown in Table 1, these observed times are in close agreement with our predicted values, thus indicating that it is valid to use the relative critical load metric and the closely related critical overload metric to evaluate the quality of assignments.

Figure 2 plots the critical overload metric for the original proportional mapping, the Robin Hood scheme and our final multi-pass scheme for augustus7 on 8 through 64 processors. The plots indicate that Robin Hood scheme can improve the assignments produced by the original proportional mapping. However, as expected, these improvements are not as substantial as the improvements from our final multi-pass scheme. Consequently, in the remainder of this section, we focus on more detailed comparisons between the quality of assignments produced by the original proportional scheme and our final multi-pass scheme.

Figure 3 (left) indicates how our scheme can improve the worst mapping generated by the original scheme. For each matrix, we select the instance with the largest value of the critical overload metric from an assignment by the pro- 

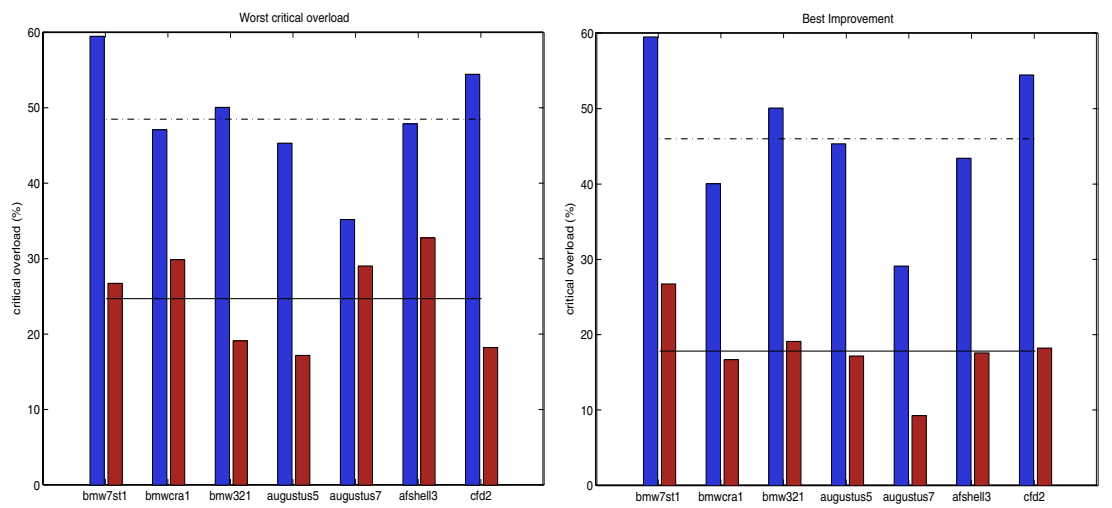

Fig. 3. Quality of assignments for worst load instances (left) and the best observed improvement instances (right); each group of two bars indicates the critical overload from the original proportional scheme and our multi-pass scheme. Average values are indicated by horizontal lines
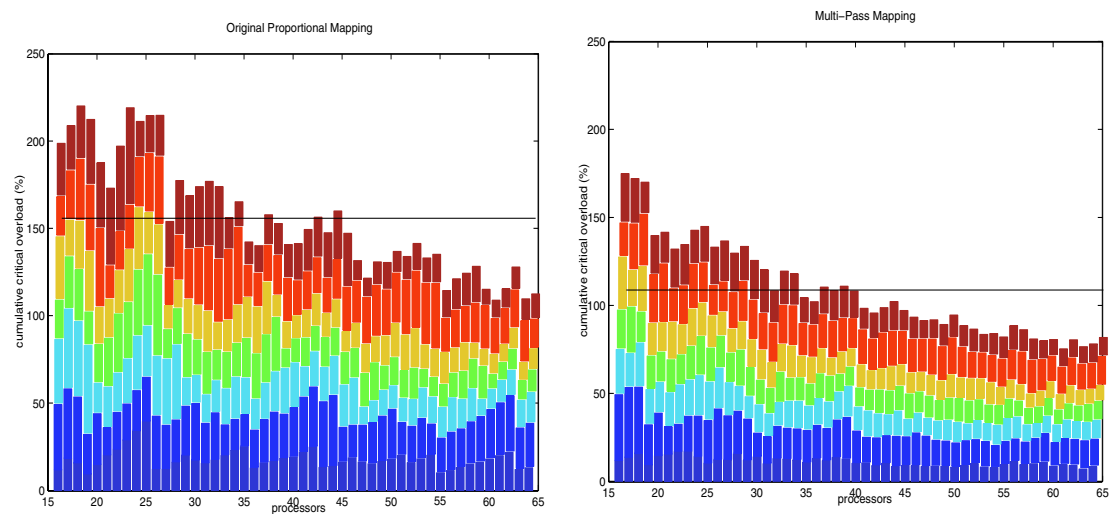

Fig. 4. Cumulative critical overload over all matrices for each processor, from assignments using the original proportional scheme (left) and from our multi-pass scheme (right). Each patch in a stacked bar represents the critical overload for one matrix. Average values are indicated by horizontal lines

portional scheme. For this instance, i.e., problem-processor pair, we show the value of the overload metric when our multi-pass scheme is used. Our multi-pass scheme successfully reduced the worst case overload from almost $60 \%$ to $27 \%$ for the bmw7st1 matrix. On average, the metric is halved from nearly $50 \%$ for the original to $25 \%$ for our multi-pass scheme. Figure 3 (right) shows the best observed improvement from our new multi-pass scheme when compared to the original mapping for each matrix in the test set. Assignments from our multipass scheme reduced the critical overload for bmw7st1 from approximately $60 \%$ to $27 \%$ and from $55 \%$ to $17 \%$ for cfd2. Additionally, on average over these in- 

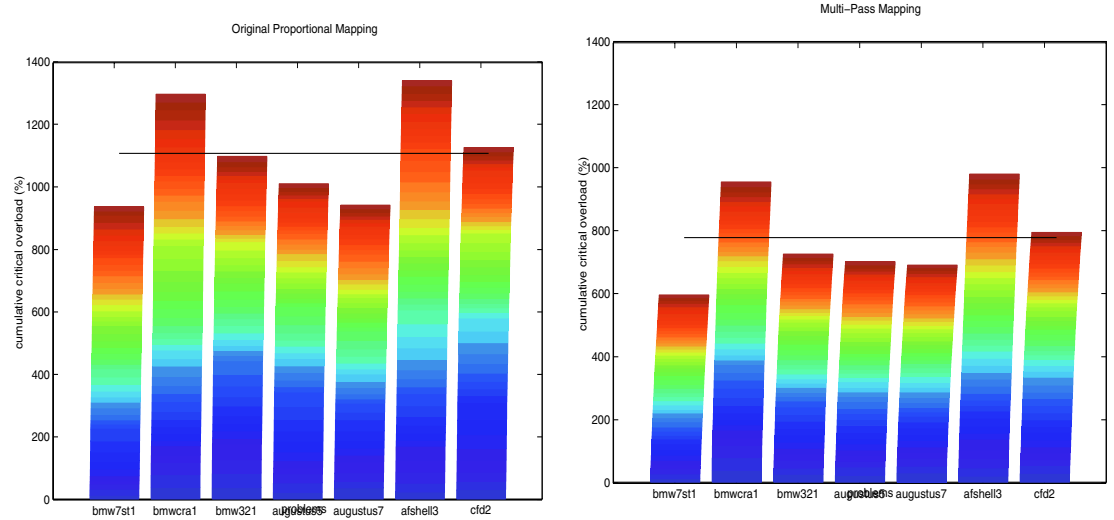

Fig. 5. Cumulative critical overload for 16 - 64 processors for each matrix, from assignments using the original proportional scheme (left) and from our multi-pass scheme (right). Each patch in a stacked bar represents the critical overload for one processor size in the range $16-64$. Average values are indicated by horizontal lines

stances, the critical overload from the proportional scheme was $46 \%$, whereas it was reduced to $17 \%$ from our multi-pass schemes.

We next consider the overall quality of the assignments produced by the original proportional scheme and our multi-pass scheme for the 7 matrices using $16-64$ processors. We now consider a cumulative form of the critical overload metric shown as a stacked bar in Figures 4 and 5. In Figure 4 each stacked bar represents the critical overload value summed over all 7 matrices for a specific number of processors. Figure 4 clearly indicates that our multi-pass scheme significantly improves the quality of mapping over all problems for the entire range of processors. On average, the cumulative critical overload is reduced from a value of approximately 150 to 100 through the use of our multi-pass scheme. Figure 5 shows the improvements for each matrix cumulatively over the range of processors; each stacked bar represents the critical overload value summed over all 49 processor sizes (from 16 - 64) for a specific matrix. Now the average value from the original scheme is approximately 1050 which is reduced to under 725 by our multi-pass mapping scheme. These results show that our multi-pass schemes are indeed effective in producing assignments that substantially improve the balance of loads among processors.

\section{Conclusions}

In this paper, we have presented a multi-pass scheme that improves workload distribution among processors in tree-structured computation. Through experiments using trees weighted to represent sparse multifrontal factorizations on distributed memory multiprocessors we show that our multi-pass mapping scheme can significantly improve the quality of assignments. 
Our scheme can be applied with a more complex weighting scheme to take into account both computation and interprocessor computation costs. Our scheme can also be used with a weighting function to model memory requirements to produce more balanced assignments. Additionally, we can compute assignments for applications where the triangular solution costs following the factorization are dominant, i.e., one factorization is followed by solutions for a sequence of right-hand-side vectors [20]. We can also extend the weighting schemes to model parallel incomplete factorization for preconditioning [18] and the subsequent application of the preconditioner using tree-structured parallel schemes.

\section{References}

1. J. Demmel, S. C. Eisenstat, J. R. Gilbert, X. S. Li, and J. W. H. Liu. A supernodal approach to sparse partial pivoting. Technical Report CSL-94-14, Xerox Palo Alto Research Center, 1995.

2. I.S. Duff. Parallel implementation of multifrontal schemes. Parallel Computing, 3:193-204, 1986.

3. A. George and J. W. H. Liu. An automatic nested dissection algorithm for irregular finite element problems. SIAM J. Numer. Anal., 15:1053-1069, 1978.

4. J. A. George and J. W. H. Liu. Computer Solution of Large Sparse Positive Definite Systems. Prentice-Hall Inc., Englewood Cliffs, NJ, 1981.

5. L. Grigori and X. S. Li. A new scheduling algorithm for parallel sparse LU factorization with static pivoting. In Proceedings of the 2002 ACM/IEEE conference on Supercomputing, pages 1-18. IEEE Computer Society Press, 2002.

6. A. Gupta, F. Gustavson, M. Joshi, G. Karypis, and V. Kumar. PSPASES: An efficient and scalable parallel sparse direct solver, 1999. See http://www-users.cs.umn.edu/\$ \$sim\$mjoshi/pspases

7. A. Gupta, V. Kumar, and A. Sameh. Performance and scalability of preconditioned conjugate gradient methods on the CM-5. In R. F Sincovec, D. E. Keyes, M. R. Leuze, L. R. Petzold, and D. A. Reed, editors, Proceedings of the Sixth SIAM Conference on Parallel Processing for Scientific Computing, pages 664-674, Philadephia, PA, 1993. SIAM Publications.

8. M. T. Heath, E. Ng, and B. W. Peyton. Parallel algorithms for sparse linear systems. SIAM Review, 33:420-460, 1991.

9. M. T. Heath and P. Raghavan. Performance of a fully parallel sparse solver. Int. J. Supercomputing Appl., 11:49-64, 1997.

10. M. T. Jones and P. E. Plassman. An improved incomplete Cholesky factorization. ACM Trans. Math. Software, 21:5-17, 1995.

11. G. Karypis and V. Kumar. METIS: Unstructured graph partitioning and sparse matrix ordering system. Technical report, Department of Computer Science, University of Minnesota, Minneapolis, MN, 1995.

12. J. W. H. Liu. The role of elimination trees in sparse factorization. SIAM J. Matrix Anal. Appl., 11:134-172, 1990.

13. J. W. H. Liu. The multifrontal method for sparse matrix solution: theory and practice. SIAM Review, 34:82-109, 1992.

14. E. Ng and B. W. Peyton. A supernodal Cholesky factorization algorithm for shared-memory multiprocessors. SIAM J. Sci. Comput., 14:761-769, 1993. 
15. A. Pothen and C. Sun. A mapping algorithm for parallel sparse Cholesky factorization. SIAM J. Sci. Comput., 14(5):1253-1257, 1993.

16. P. Raghavan. DSCPACK: Domain-Separator Codes for the parallel solution of sparse linear systems, 2002. Software for solving sparse linear systems on multiprocessors an d NOWs using C and MPI. Package has a two stage parallel nested dissection, higher-level BLAS, fast repeated solves and an easy t o use parallel interface. See http://www.cse.psu.edu/Dscpack

17. P. Raghavan, K. Teranishi, and E. Ng. Scalable parallel preconditioning with incomplete factors. In Proceedings of the Seventh SIAM Conference on Applied Linear Algebra. 2000.

18. P. Raghavan, K. Teranishi, and E. Ng. A latency tolerant hybrid sparse solver using incomplete Cholesky factorization. Numerical Linear Algebra, 10:541-560, 2003.

19. Y. Saad. Iterative Methods for Sparse Linears Systems. PWS Publishing Co., Boston, MA, 1996.

20. C. Yang, P. Raghavan, L. Arrowood, B. Sumpter, and D . Noid. Large-scale normal coordinate analysis on distributed memory mu ltiprocessors and nows. Int. J. Supercomputing Appl., 1(4):409-424, 2002. 